\title{
Review of: "Chemical durability of Gd2Zr2O7 transparent ceramics under different $\mathrm{pH}$ conditions"
}

\author{
Shaoyun $\mathrm{Pu}^{1}$ \\ 1 Southeast University
}

Potential competing interests: The author(s) declared that no potential competing interests exist.

In this study, the authors attempt to evaluate the leaching behavior of Gd2Zr2O7 ceramics under different $\mathrm{pH}$ values to simulate the deep geological landfill environment. The research and reported results in this manuscript are interesting, and the proposed conclusions are valuable for the nuclear waste immobilization. Nevertheless, there are still some minor problems in the paper, which need to be further improved. After revision, the paper can be accepted for publication. The existing problems are as follows: 1. In the GIXRD analysis, the authors have analyzed cell parameters and the results exhibit lattice shrinkage in the leached samples. To improve the readability, the authors should explain the lattice shrinkage phenomenon.

2. There are several English formatting and grammar errors in the manuscript. Please check and correct them.

3. The accuracy of the relevant $\mathrm{pH}$ values in the manuscript and in the figure should be harmonized.

4. Reference 24 and Reference 29 have errors in the document format.

5. In line 28, change "with long half-life" to "with a long half-life".

6. In line 97 , the issue regarding the spaces before the numbers and symbols should be revised.

7. In line 141, insert "the" before "leaching experiment".

8. In all tables, the number of decimal places for the relevant $\mathrm{pH}$ values should be the same as in the manuscript.

9. Authors should be aware that the use of spaces in the manuscript should be standardized, and improper use should be adjusted. 
